Meta

Journal des traducteurs

Translators' Journal

\title{
La traduction juridique et son enseignement : aspects théoriques et pratiques
}

\section{Jean-Claude Gémar}

Volume 24, numéro 1, mars 1979

La traduction juridique

URI : https://id.erudit.org/iderudit/002870ar

DOI : https://doi.org/10.7202/002870ar

Aller au sommaire du numéro

Éditeur(s)

Les Presses de l'Université de Montréal

ISSN

0026-0452 (imprimé)

1492-1421 (numérique)

Découvrir la revue

Citer cet article

Gémar, J.-C. (1979). La traduction juridique et son enseignement : aspects théoriques et pratiques. Meta, 24(1), 35-53. https://doi.org/10.7202/002870ar d'utilisation que vous pouvez consulter en ligne. 


\section{La traduction juridique et son enseignement : aspects théoriques et pratiques}

La traduction juridique ne constitue qu'une des données particulières du problème plus général que pose la traduction proprement dite. L'enseignement de cette discipline est au cour du débat opposant depuis plus de vingt ans les linguistes pour lesquels il est possible de faire passer dans la langue d'arrivée (LA) l'intégralité du message exprimé dans la langue de départ (LD) et ceux, tout aussi nombreux et convaincus, qui estiment impossible l'acte traductionnel puisque chaque langue est un instrument social de communication, le produit original d'une culture, d'une mentalité et d'un milieu nécessairement uniques ${ }^{1}$.

À la question que posait déjà Vinay en 1957 : «Peut-on enseigner la traduction ${ }^{2}$ ?», on peut apporter trois types de réponses. Depuis la publication de la Stylistique comparée du français et de l'anglais, le monde de la traduction est doté de l'ossature méthodologique nécessaire pour aborder de manière pratique mais formelle la plupart des problèmes que pose l'opération de transfert à toute personne qui se propose de rendre dans LA les éléments contenus dans LD, quelle que soit la combinaison linguistique envisagée, du moins entre les principales langues de culture occidentale ${ }^{3}$.

Le premier type de réponse est celui que proposent Vinay et Darbelnet qui répondent par l'affirmative à la question posée plus haut. Vinay, notamment, tout en signalant les difficultés que présente un tel enseignement, en reconnaît la * nécessité » en situation de bilinguisme et lui attribue même des fonctions supérieures de véhicule de communication et de culture ${ }^{4}$.

Cette opinion est loin de faire l'unanimité chez les linguistes. Georges Mounin ${ }^{5}$ remonte à Saussure, cite Martinet, Bloomfield, Harris et Hjelmslev comme autorités pour formuler une objection théorique, fondée sur le sens de l'énoncé

1. Voir l'étude de Pierre Guiraud consacrée aux «Fonctions secondaires du langage », et plus particulièrement \& Langage, individu et société $*$, le Langage, 25 e volume" de l'Encyclopédie de la Pléiade, Paris, Gallimard, 1968, p. 459 et suiv.

2. Journal des traducteurs, II, 4, 1967, p. 141 et suiv.

3. Voir Georges Mounin, les Problèmes théoriques de la traduction, Paris, Gallimard, 1963, 290 p., rééd. 1976, dans la coll. TEL, et l'étude consacrée à̀ cette question au chap. XIII, "L'ethnographie est une traduction', p. 227 et suiv.; E.A. Nida, clinguistics and Ethnology in Translation Problems *, Word, 1945, no 2, p. 194-208, cité par Mounin, op. cit., p. 240.

4. Georges Mounin, les Problèmes théoriques de ta traduction, op. cit., p. 147.
5. Ibid., p. 21-40. 
linguistique, "soit contre la légitimité, soit même contre la possibilité de toute traduction ${ }^{6} \$$, mais admet néanmoins que ces théories linguistiques modernes « n'ont entamé, cependant, ni la légitimité théorique, ni la possibilité pratique des opérations de traduction ${ }^{7} \gg$.

Toutefois, par-delà la diversité des langues, l'objectif du linguiste est de retrouver le langage, d'aller en quelque sorte des langues - plus petit dénominateur commun linguistique - au langage - plus grand dénominateur commun

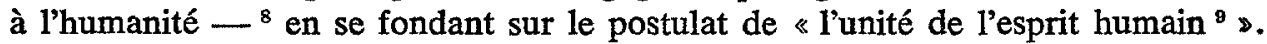
Cette vue est partagée par un nombre de plus en plus grand de linguistes dans les rangs desquels nous pourrions faire figurer les pragmatistes, et ils sont légion, que sont les traducteurs et interprètes, les fonctionnaires, techniciens et autres spécialistes, bilingues ou polyglottes, les enseignants spécialisés en langues vivantes ou mortes, pour ne citer que quelques catégories professionnelles. Le mérite de Vinay et Darbelnet est d'avoir compris, au lieu de s'enliser dans de vaines querelles d'écoles linguistiques, la nécessité d'établir un répertoire assez complet des problèmes de traduction et proposé une méthodologie visant à les résoudre 《en situation», à partir d'exemples concrets.

A cela on rétorquera que leur méthode a pris de l'âge et qu'elle est souvent contestée. Contestée, certes, mais dépassée ? Non pas certes ! Le fond, lui, reste le même si l'exposé accuse un certain retard par rapport à l'actualité, à une époque où l'on éprouve quelque peine à suivre le fil de l'événement. La conclusion de Mounin citée plus haut ne fait que renforcer le bien-fondé de leur thèse, en attendant que les théories inlassablement annoncées (mais elles se font attendre!) ne viennent prendre un relais non moins inlassablement signalé à l'horizon de la linguistique, mais toujours aussi éloigné.

Laissons encore à Georges Mounin le soin de conclure cette introduction : - L'activité traduisante pose un problème théorique à la linguistique contemporaine : si l'on accepte les thèses courantes [...] on aboutit à professer que la traduction devrait être impossible. Mais les traducteurs existent, ils produisent, on se sert utilement de leur production. On pourrait presque dire que l'existence de la traduction constitue le scandale de la linguistique contemporaine. Jusqu'ici l'examen de ce scandale a toujours été plus ou moins rejeté ${ }^{10}$.

La traduction est donc possible et, en outre, on peut même l'enseigner.

Il reste que dans l'esprit de beaucoup un malaise, un certain doute subsiste dès lors que la traduction est en cause. Le binôme TRADUCTION = TRAHISON dont on affuble si souvent et si commodément le traducteur n'est qu'un exemple parmi tant d'autres. Telle Hester Prynne, il est appelé à porter son fardeau d'humiliation et de réprobation. Un exemple tiré de la presse hebdoma-

\footnotetext{
6. Georges Mounin, les Problèmes théoriques de la traduction, op. cit., p. 21.

7. Ibid., p. 40.

8. Frédéric François, Le langage et ses fonctions *, le Langage, op, cit., p. 3-5.

9. Lazar Focsaneanu, \&Les langues comme moyen d'expression du droit international 》, Annuaire français de droit international, XVI, 1970, Paris, C.N.R.S. 1971, p. 256 et suiv.

10. Georges Mounin, les Problèmes théoriques de la traduction, op. cit., p. 8. C'est moi qui souligne.
} 
daire, à propos d'un événement de l'actualité, illustre clairement notre propos : * Ne voient-ils pas [Bossuet et Péguy] que si le Christ a prêché dans l'Empire, c'est au prix d'une traduction, c'est-à-dire d'une trahison ${ }^{11}$ ? »

Cela étant dit, la démarche suivie en traduction générale s'applique-t-elle également à la traduction juridique ?

À l'évidence, les principes restent les mêmes et les mécanismes de la traduction jouent de la même manière; que le texte considéré soit d'ordre général, scientifique ou technique, il s'agit toujours de faire passer un message pour qu'il soit compris du destinataire. C'est le postulat de Vinay, à savoir que \& le sens seul compte », c'est-à-dire le résultat en définitive, puisque l'on ne traduit pas pour comprendre mais pour faire comprendre et que « tous les moyens sont bons en traduction pourvu que le sens soit respecté ${ }^{12}$ \%.

Un tel postulat peut-il s'appliquer à la traduction juridique et à son enseignement? Oui, si l'on pose en principe que cette discipline fait intervenir des techniques d'apprentissage fort comparables à celles qui sont utilisées dans l'enseignement des langues de spécialité ou de la traduction dite « technique ». Les problèmes méthodologiques que peut poser l'enseignement de la traduction juridique sont à priori les mêmes que les « obstacles linguistiques » dont parle Mounin et correspondent grosso modo aux difficultés reconnues et classées par Vinay et Darbelnet. Quel que soit le type de traduction envisagé, les problèmes d'ordre lexical ou syntaxique demeurent.

Donc, en résumé, au sens « technique » du terme, on ne peut dissocier la traduction juridique des autres formes de traduction.

Ici s'arrête le parallélisme des démarches car, sur le plan du fond, de la matière proprement dite, cette branche de la traduction s'écarte considérablement des méthodes, techniques et préoccupations propres à la traduction « générale * et même aux autres formes de traduction technique.

En outre, son enseignement n'est pas sans présenter un certain nombre de difficultés spécifiques qui en font véritablement une discipline à part entière, originale par son objet, très complexe par la diversité des données et la grande variété des domaines qu'elle regroupe.

\section{Problématique de la traduction juridique}

Le truisme le plus souvent entendu à propos du droit est celui qui fait état de sa fonction sociale, ubi societas, ibi jus. Le droit est un phénomène social, le produit d'une culture, mais cette évidence revient à poser en préalable un principe fondamental, celui de la norme qui s'appuie sur les trois piliers du droit que sont la famille, la propriété et le contrat édifiés sur la base d'une réglementation dont l'aboutissement logique, normal, est le caractère contentieux de la règle juridique.

11. Jean Grosjean, le Nouvel Observateur, no 685, 26 déc. 1977 - 1er janvier 1978, p. 61,

12. Journal des traducteurs, op. cit., p. 141. 
Ce seul postulat confère à la traduction juridique, donc à son enseignement, une valeur tout à fait spécifique que l'on ne retrouve dans aucun autre domaine de la traduction car, pour qu'intervienine le due process of law cher au droit anglo-américain, il convient de définir le ou les concepts normatifs applicables en l'espèce. Or, contrairement à une autre idée reçue en la matière, la norme ne présente pas le caractère immuable, figé que l'on pourrait croire ; elle est essentiellement mouvante en raison du contexte social qui l'a créée et ne saurait donc, à l'instar de la règle juridique, s'appliquer en dehors du cadre étroit des États ${ }^{13}$ : Il suffit pour s'en convaincre de voir comment les différentes conceptions normatives envisagent la notion de «bonnes mours » par exempe, aux États-Unis, en Italie, en Turquie ou au Japon, ou encore de comparer le concept de rationabili parte bonorum (the « reasonable » share of the goods) dont parle Blackstone ${ }^{14}$ à celui du «bon père de famille » auquel le Code civil fait encore allusion ${ }^{15}$. Seule la sociologie pourrait éclairer le juriste et conférer tout leur sens aux facteurs sociaux qui ont conditionné la formation objective de normes telles que le reasonable man ou le «bon père de famille». Il n'est d'ailleurs pas exclu que leur valeur sémantique réelle soit, en fin de compte, identique ${ }^{16}$, mais la démarche qui a présidé à leur élaboration est, elle, le produit spécifique de la société qui les a créées ${ }^{17}$.

La norme juridique implique comme conséquence logique, et pour ainsi dire inéluctable, l'obligation juridique. Qui dit obligation dit contrainte, c'est-à-dire sanction ${ }^{18}$. Là réside la spécificité du texte juridique : son caractère contraignant. Cette prémisse fondamentale confère toute sa signification à l'apprentissage de la traduction juridique dont elle conditionne les techniques, la démarche et la finalité, établissant sans équivoque son unicité, son statut de discipline à part entière.

\section{Spécificitê de la traduction juridique}

Cinq critères principaux, formant une typologie non exhaustive, constituent le fondement épistémologique de la traduction juridique. Ce sont, dans l'ordre d'importance que nous leur reconnaissons, le caractère normatif (ou contraignant) du texte juridique, le discours (ou langage) du droit, la diversité sociopolitique des systèmes juridiques, tous éléments conditionnant le problème de la documentation juridique auquel il faut lier la nécessité d'une approche pluridisciplinaire du droit et, partant, de son enseignement.

Ce qui est vrai de l'enseignement du droit, l'est également de l'enseignement de la traduction juridique.

\footnotetext{
13. Voir notamment sur ce point Ch. Perelman, Logique juridique, Paris, Dalloz, 1976, no 90 , p. $165-166$.

14. Commentaries on the Laws of England, ed. by W.C. Jones, Baton Rouge, Clairtor's, 1976 Reprint, Book II, p. 1409.

15. Art. 601 du Code civil par ex.

16. "L'honnête homme » du siècle des lumières n'est sans doute pas très éloigné du reasonable man anglais.

17. Voir à ce propos E.S. de la Marnierre, Eléments de méthodologie juridique, Paris, Librairie du Journal des notaires et des avocats, 1976, no 12, p. 29-31.

18. Cette question de la norme et de son schéma d'interprétation a fait l'objet d'une analyse poussée et pénétrante de la part de Hans Kelsen dans son ouvrage fondamental Reine Rechtslehre, publié en français (dans une excellente traduction) sous le titre Théorie pure du droit, Paris, Dalloz, 1962, $496 \mathrm{p}$.:
} 
Le cadre du présent article ne nous permettant pas de développer notre argumentation de manière exhaustive, nous nous bornerons à présenter les grandes lignes de ce qui fera l'objet de développements ultérieurs et ponctuels.

\section{LE CARACTÈRE NORMATIF DU TEXTE JURIDIQUE}

Le postulat sur lequel la majorité des juristes s'accordent pour une fois avec les linguistes est celui qui reconnaît au texte juridique un caractère contraignant qui lui est propre. Cette notion de contrainte est généralement absente des études consacrées à la traduction. Il y est pourtant fait allusion d'une certaine manière à propos des «servitudes ${ }^{19} »$, au sens de Vinay et Darbelnet, et de la planification linguistique, encore que le taux des échecs essuyés par les commissions linguistiques soit assez élevé pour parler de la relativité de cette forme de contrainte.

Rien de tel en traduction juridique où celle-ci doit s'aligner sur la règle établie par la constitution, la loi ou le règlement, respecter l'obligation portée dans le contrat et s'incliner devant la décision du tribunal. Cette contingence est un préalable à toute approche objective du droit.

On rétorquera que la traduction juridique peut porter sur d'autres textes que ceux qui présentent un caractère contraignant, une servitude juridique, notamment lorsqu'il ne s'agit que d'informer, renseigner le destinataire. Les conséquences, la portée de l'acte traductionnel seront, selon le cas, fort différentes, l'erreur n'entraînant dans le second qu'un minimum d'effets, alors que dans le premier cas, surtout si le texte relève du domaine public, les retombées peuvent être lourdes de conséquences ou avoir des effets imprévus. L'exemple que donne Madeleine Sauvé, le grammairien de l'Université de Montréal, à propos de la ponctuation, est particulièrement édifiant et devrait faire réfléchir l'apprenti traducteur :

Le 24 février 1959, la cour d'appel de Dijon a réglé un litige ayant pour enjeu 40 milliards ${ }^{20}$, qui reposait sur l'interprétation d'une virgule dans l'article 9 de la loi du 24 mars 1952 sur les sociétés de crédit différé ${ }^{21}$.

On pourrait donc en déduire, et l'on n'aurait pas tout à fait tort, que la marge de manœuvre du traducteur, à fortiori celle de l'enseignant, est étroite et qu'il est tenu de suivre aveuglément les contraintes, l'économie de LD.

C'est le problème qui se pose, sous forme de cas de conscience, au traducteur juridique. Doit-il traduire selon son ccur ou selon sa raison le texte qui lui est

19. J.-P. Vinay et J. Darbelnet, Stylistique comparée du français et de l'anglais, Paris, Didier, 1971, paragr. 11, p. 31. L'économie des servitudes linguistiques suit un cheminement très différent et son caractère contraignant est très faible. L'exemple de l'imparfait du subjonctif cité par les auteurs est parfaitement révélateur à cet égard. Nul n'est tenu par la loi (ce n'est qu'une convention) d'employer le subjonctif mais \&nul n'est censé ignorer la loi » et «L'ignorance de la loi chez une personne qui commet une infraction n'excuse pas la perpétration de cette infraction » (art. 19 du Code criminel). Toute la différence est là.

20. Il s'agit ici de francs français, soit environ 100 millions de dollars.

21. Observations grammaticales et terminologiques, Secrétariat général de l'Université de Montréal, Fiche no 86, oct. 1977, p. 4 ; cette citation est extraite du Grand Larousse de la langue française, t. V, 1975, s.v., Ponctuation. 
soumis, compte tenu du caractère obligatoire qu'est appelée à revêtir la traduction? Cette question se pose avec une très grande force si la loi (fédérale, communautaire, nationale ou internationale) confère l'égalité de statut à deux ou à plusieurs langues officielles. Les quelques exemples qui suivent apporteront sans doute un élément de réponse à cette question. Ils sont tirés de textes juridiques canadiens, documents officiels et publics, rédigés en anglais et traduits en français selon deux conceptions très différentes.

\section{Ex. 1}

(3) Subject to subsection (4), a person is not justified for the purposes of subsection (1) in using force that is intended or is likely to cause death or grievous bodily harm unless he believes on reasonable and probable grounds that it is necessary for the purpose of preserving himself or any one under his protection from death or grievous bodily harm.

(4) A peace officer who is proceeding lawfully to arrest, with or without warrant, any person for an offence for which that person may be arrested without warrant, and every one lawfully assisting the peace officer, is justified, if the person to be arrested takes flight to avoid arrest, in using as much force as is necessary to prevent the escape by flight, unless the escape can be prevented by reasonable means in a less violent manner. $1953-54$, c. 51 , s. 25.

\section{Ex. 2}

2. The purpose of this Act is to extend the present laws in Canada with respect to matters coming within legislative authority of the Parliament of Canada to give effect to the principles

a) that every individual should have an equal opportunity with other individuals to make for himself or herself the life that he or she is liable and wishes to have, consistent with his or her duties and obligations as a member of Society, without being hindered in or prevented from doing so by discriminatory practices based on race, national or ethnic origin, colour, religion, age, sex or marital status, or conviction for an offence for which a pardon has been granted; and

b) that the privacy of individuals should be protected to the greatest extent consistent with public order and well-being;
(3) Subordonnément au paragraphe (4), une personne n'est pas justifiée, aux fins du paragraphe (1), d'employer la force avec l'intention de causer, ou de nature à causer la mort ou des lésions corporelles graves, à moins qu'elle n'estime, pour des motifs raisonnables et probables, que cette force est nécessaire afin de se protéger elle-même ou de protéger toute autre personne sous ses soins, contre la mort ou contre des lésions corporelles graves.

(4) Un agent de la paix qui procède légalement à l'arrestation, avec ou sans mandat, d'une personne pour une infraction au sujet de laquelle cette personne peut être appréhendée sans mandat, ainsi que toute personne aidant légalement l'agent de la paix, est justifiable, si la personne qui doit être appréhendée s'enfuit afin d'éviter l'arrestation, d'employer la force nécessaire pour empêcher cette fuite, à moins que l'évasion puisse être empêchée par des moyens raisonnables d'une façon moins violente. 195354 , c. 51 , art. 2522 .

2. La présente loi a pour objet de compléter la législation canadienne actuelle en donnant effet, dans le champ de compétence du Parlement, aux principes suivants :

a) tous ont droit, dans la mesure compatible avec leurs devoirs et obligations au sein de la société, à l'égalité des chances d'épanouissement, indépendamment des considérations fondées sur la race, l'origine nationale ou ethnique, la couleur, la religion, l'âge, le sexe, la situation de famille ou l'état de personne grâciée ;

b) le droit à la vie privée doit être protégé dans toute la mesure compatible avec l'ordre public et le bien-être général.

22. Code criminel, art. 25(3) et (4). 
6. A permit officer who receives from a resident of Canada an application for an export permit shall issue the permit forthwith if the person applying for the permit establishes to the satisfaction of the permit officer that the object in respect of which the application is made

a) was imported into Canada within the thirty-five years immediately preceding the date of the application and was not exported from Canada under a permit issued under this Act prior to that importation;

b) was loaned to an institution or public authority in Canada by a person who was not a resident of Canada at the time of the loan was made; or

c) is to be removed from Canada for a purpose prescribed by regulation for a period of time not exceeding such period of time as may be prescribed by regulation for the purposes of this paragraph.
6. L'agent délivre sans délai une licence à tout résident qui le convainc de ce que l'objet visé dans sa demande

a) a été importé au Canada au cours des trente-cinq ans précédant la date de la demande sans avoir été auparavant exporté du Canada sous le couvert d'une licence, d'une licence générale ou d'un permis, délivré en vertu de la présente loi ;

b) a été prêté à un établissement ou à une administration, sis au Canada, par une personne qui était alors un nonrésident; ou

c) serait sorti du Canada à des fins et pour une durée limite conformes au réglement d'application du présent alinéa ${ }^{23}$.

On voit à quels abus peuvent conduire les «contraintes » imposées par le texte juridique de LD dans l'exemple $\mathrm{n}^{\circ} 1$ et à quels heureux résultats on peut arriver si l'on s'applique à respecter les servitudes de LA, comme dans les exemples donnés en 2 . Or, le caractère contraignant des textes, dans les deux cas, est identique, que la traduction soit calquée sur LD ou non! Encore s'agit-il ici des textes de loi dont le caractère public fait obligation au législateur d'introduire des considérations de rigueur et de clarté souvent absentes des textes à caractère privé, tels les contrats dans lesquels il n'est pas rare de trouver des clauses de 30 , 40 lignes et plus, sans même un point-virgule ! Les contraintes qui s'exercent sur ce dernier genre de textes ont, en théorie, une portée beaucoup plus faible que dans le premier cas et la question de l'interprétation du texte joue donc en l'occurrence un rôle moins important. Nous ne nous étendrons pas sur ce point, fondamental, qu'est la notion d'interprétation en common law, qui fait l'objet d'un autre article du présent numéro ${ }^{24}$.

Il reste que le texte juridique, notamment en droit public (et surtout en droit international public où interviennent les relations diplomatiques), impose au traducteur de suivre les rails d'une logique dont il ne saurait s'écarter sans risquer de déclencher une réaction dont les effets lui échapperont dans la plupart des cas : c'est l'effet «serendip \& qui frappe l'imprudent qui s'expose à l'ire de Thémis.

Cette dernic̀re considération nous amène tout naturellement au deuxième terme de notre postulat, celui du langage du droit.

\section{LE LANGAGE DE THEMIS}

L'unicité de la traduction juridique découle également de la spécificité de la langue du droit par laquelle s'exprime la règle. L'étude de cette langue revêt

23. Bill C-72, art. 2 ; Bill C-23, art. 6.

24. Voir à ce sujet l'article de Marie Lajoie \& L'interprétation judiciaire des textes législatifs bilingues \& qui eclaire une question très controversée. 
une importance particulière puisque de son interprétation dépendent l'application normative et la mise en œuvre de la règle juridique.

C'est naturellement le sens et non la forme d'un texte qu'il faut rendre. Le traducteur, pour ce faire, dispose des ressources terminologiques qu'offre la langue de spécialité aux utilisateurs pour décrire en termes non équivoques une réalité, une notion que le vocabulaire courant cerne avec difficulté. Le droit ne déroge pas à cette règle, car il possède un vocabulaire particulièrement riche qui comprendrait selon les pays entre 10000 et 20000 termes ${ }^{25}$, d'où une grande complexité lexicale que multiplie encore la traduction puisque ce n'est pas une langue mais deux, et parfois davantage, que le traducteur est amené à traiter. C'est donc sur un fonds terminologique variant entre 20000 mots au moins et \pm 40000 que s'exerce l'activité traduisante dans le domaine du droit. C'est dire la difficulté de la tâche qui attend le traducteur appelé, nécessité oblige, à se transformer en comparatiste.

Chaque domaine de spécialité dispose de son vocabulaire, de son jargon diront certains, pour qualifier avec le plus de précision possible, dans une perspective de stabilité sémantique et de certitude scientifique sans lesquelles ses activités ne pourraient se dérouler normalement et dans la continuité, les techniques, mécanismes, notions et autres phénomènes propres à ce domaine.

Sans aller jusqu'à dire que 1a traduction juridique se résumerait à une question de langage, celui de la «technique » juridique, il est néanmoins impossible de ne pas tenir compte de sa terminologie et il faut admettre en préalable le postulat de Gény, selon lequel «prise dans son ensemble, la technique juridique aboutit, pour la plus grande part, à une question de terminologie ${ }^{26} 》$.

Or, rien n'est plus malaisé que de tenter de cerner la réalité de la terminologie du droit qui, contrairement à une autre idée reçue, n'est pas aussi évidente qu'il y paraît. Le langage du droit est-il plus technique que scientifique ou inversement ? Existe-t-il à proprement parler un langage du droit? Ne devrait-on pas plutôt parler de langage « juridique » ou encore des langages du législateur, du juge, du théoricien, du praticien, dont les racines sont profondément ancrées dans le tronc commun à tous qu'est la langue vulgaire, mis à part quelques exceptions bien spécifiques, quelques emprunts au latin ou à d'autres langues ?

Nous ne nierons pas l'existence d'une terminologie juridique, qui est incontestable, mais nous contesterons son caractère spécifique. En effet, en dehors des mathématiques ou de la physique, les deux disciplines le plus souvent citées comme modèles du langage technique «parfait », c'est-à-dire dont le vocabulaire exclut toute ambiguïté, quelle discipline peut se targuer de posséder un vocabulaire qui lui soit propre, qui ne prête à aucune équivoque, dont les termes ne revêtent qu'un sens possible, excluant toute ambiguïté sémantique. Ce n'est pas dans les disciplines qui fondent leur existence sur une conception particulière de l'ordre social

25. Chiffres avancés par Aurel David, chercheur au C.N.R.S., lors d'une conférence donnée à la Faculté de droit de l'Université de Montréal le 21 oct. 1976.

26. François Gény, Science et technique en droit privé positif, Paris, Sirey, 1921, t. III, no $255, \mathrm{p}, 456$. 
qu'il faut chercher cette absence de "naïveté » du langage, au sens que lui donne Aurel David; les juristes seraient donc «naïfs car ils n'ont pas de langage fondamental sur quoi bâtir une science avec toute la rigueur que confère un vocabulaire essentiel.

Prenons l'exemple du mot «droit». De très nombreux juristes s'efforcent de donner à ce terme une définition précise, ce qui nous apporte une grande diversité de réponses à la question «Qu'est-ce que le droit? »Consultez plutôt 4 ou 5 dictionnaires qui vous apprendront que le mot clé de cette immense discipline, celui qui devrait être le mieux défini, précisé, a un sens fort déroutant pour le profane, et chacun y va de sa définition. Encore ne parlons-nous pas des définitions qu'en donne chaque idéologie, souvent dans un seul et même pays. À fortiori, on peut imaginer ce que peut donner la consultation de dictionnaires de droit unilingues d'autres pays pour faire le lien entre deux systèmes, établir des comparaisons entre une tradition occidentale et une tradition orientale par exemple. Mais n'anticipons pas sur notre propos.

Un autre bon exemple est celui du mot « équité ». Peu de juristes définiront cette notion essentielle du droit en termes rigoureusement semblables. Nous pourrions multiplier ainsi les exemples. L'incertitude du vocabulaire découle généralement du flou des concepts et le traducteur, n'ayant aucune prise sur ceux-ci, doit s'en tenir au vocabulaire. C'est ici qu'apparaît la difficulté de sa tâche dans toute sa complexité, la polysémie des termes juridiques lui posant des chaussetrappes à chaque coin de phrase. La fameuse clarté de la langue française, la rigueur de la rédaction des lois ne seraient-elles qu'un mythe, tenace il est vrai ${ }^{27}$ ? Cela est fort probable. Il n'en faut pas conclure pour autant que le langage de la common law présente toutes les garanties déniées au droit de tradition romanogermanique : le péché est ici universel et les vertus de l'un ne peuvent racheter les vices de l'autre. En vérité, tous les langages du droit pèchent par cette absence de vocabulaire essentiel et les nombreuses tentatives de clarification et de précision $\mathrm{du}$ droit français et du droit anglo-américain semblent, du moins pour l'instant, vouées à l'échec tant qu'elles ne seront pas appuyées par l'intervention concrète des organismes de planification linguistique ${ }^{28}$, quoique les États-Unis, avec les partisans de la Plain Language Law, aient enregistré de nets succès puisque 19 États (dont New York, la Californie, l'Ohio, l'Illinois, etc.) ont adopté une loi de ce genre, ou songent à le faire.

Le traducteur juridique se trouve donc placé dans une incertitude dont le juriste serait le principal responsable. Le législateur ne s'est pas toujours montré à la hauteur des grandes tâches que l'Histoire lui a confiées. L'enseignement d'une discipline dont le fondement terminologique est aussi vaste que flottant ne peut

27. Voir sur cette question l'intéressant article de Roger Nerson, « Exercices de vocabulaire », in Mélanges Voirin, Paris, L.G.D.J., 1967, p. 603 et suiv.

28. Sur cette question, voir en particulier les ouvrages suivants : le Langage du droit, de J.-L. Sourioux et P. Lerat, Paris, P.U.F., 1975, 133 p.; le Langage de la justice pénale, de L.-M. Raymondis et M. Le Guern, Paris, Editions du C.N.R.S., 1976, 201 p. ; le Langage du droit, collectif, Paris, Sirey, coll. "Archives de philosophie du droit», t. XIX, 1974, 556 p.; Philosophie du droit, de Michel Villey, Paris, Dalloz, 1975, 242 p., nos 4 et 129 ; Simplify Legal Writing, by Elliott L. Biskind, New York, Arco, 1975,177 p. 
qu'accroître son désarroi entretenu par la confusion des divers langages venus s'ajouter au langage du droit depuis que les Romains lui ont donné ses lettres de noblesse, la forme et le contenu qu'on lui connaît.

Comment pourrait-il en être autrement, d'ailleurs, devant la dualité des langues juridiques et les débordements auxquels assistent, impuissants, les consommateurs de services juridiques. La règle ejusdem generis n'arrange rien en droit anglo-américain et le fait de multiplier les termes «précis » à la suite les uns des autres n'éclaire en rien l'utilisateur. Dans les baux commerciaux on voit de plus en plus souvent de net list, de net net list et de net net net list pour signaler que les frais sont plus ou moins dégressifs! Sans doute est-ce le résultat d'une tendance que dénoncent les auteurs d'une étude faite pour le compte de la Commission de réforme du droit au Canada : «The legal profession's penchant for using too many words is a result of trying to be as precise as possible ${ }^{29} . \gg$ On se passerait parfois d'un tel souci de précision...

Toutes ces considérations font du langage du droit un domaine à part que certains n'hésitent pas à qualifier de secteur «maudit » de la terminologie en raison des grandes difficultés qu'il présente, tant pour le rédacteur que le traducteur ou le terminologue, entre autres sur le plan méthodologique. Dans quelle catégorie, en effet, classer des termes comme acte, accord, droit, équité, crime, infraction, motif, etc. ? Dans celle des termes techniques ou scientifiques? Ou dans le corpus de la langue courante, car ils ne peuvent passer pour des mots uniquement juridiques. Le droit est un colosse dont les pieds sont d'argile.

Cette ambiguïté du vocabulaire lui confère un caractère unique en traduction et contribue à faire de la traduction juridique une discipline à part. Mais le langage du droit n'est que la manifestation concrète, l'émanation de l'ordre social qui l'a engendré. Il est le produit de tout un système socio-politique original qui constitue le troisième terme de notre postulat.

\section{DIVERSITÉ DES SYSTÈMES JURIDIQUES}

La seule vraie grande difficulté, mais elle est de taille, que présente la traduction juridique procède de la variété et de la diversité des systèmes juridiques en présence.

Ces différences découlent principalement des diverses définitions du droit selon les pays et les mœurs et de la finalité du droit, l'objet vers lequel il tend. Des cinq critères que nous avons reconnus comme fondement épistémologique de la traduction juridique, celui-ci est le plus évident parce qu'il se fonde sur une réalité directement perceptible pour l'observateur appelé à franchir les frontières juridiques que des siècles d'application ont édifiées autour des divers Etats, que ceux-ci participent de la grande famille romano-germanique, de la common law, du droit musulman ou des droits extrême-orientaux.

On connaît les difficultés que pose la traduction juridique, dont le but est de faire passer de LD dans LA des notions parfois véritablement inconnues de

29. Language and Jury Instructions, by E.R. Myers and C.S. Jones, Ottawa, Law Reform Commission of Canada, 1978 (à paraître). 
cette dernière, dans une situation « simple » comme en connaissent les pays qui ne possèdent qu'une langue officielle, ou plus complexe dans ceux qui en possèdent deux (et le Canada n'a pas l'exclusivité dans ce domaine); que dire des organisations où cohabitent 4,5 ou 6 langues officielles ? Les organisations internationales (O.N.U.) et régionales (C.E.E.) ont le douteux privilège de devoir fonctionner en plusieurs langues de statut souvent équivalent. Les difficultés sont alors fonction du nombre de systèmes en présence. Dans certains cas, les différences que présentent certains d'entre eux (cas de la C.E.E., par exemple) sont minimes et il est sans doute plus facile de traduire en français des textes rédigés en allemand, espagnol, italien, portugais ou hollandais que de traduire de l'anglais vers le français ou du chinois vers le français ou l'anglais. La difficulté n'est pas directement proportionnelle à la distance qui sépare les systèmes entre eux, la Manche étant un fossé parfois plus difficile à franchir que l'Atlantique ou la Méditerranée puisque la plupart des pays d'Amérique latine et certains pays du Proche-Orient ont un système juridique qui s'inspire directement des droits romano-germaniques.

Bien qu'il ne soit pas dénué d'intérêt d'étudier les caractéristiques des différents types de droit auxquels le traducteur peut avoir affaire, une telle étude déborderait du cadre du présent article. Nous ne retiendrons ici que les aspects intéressant la traduction de l'anglais au français, soit les deux familles juridiques que représentent la tradition civiliste et le droit anglo-américain. Elles présentent des différences fondamentales quant au fond et dans la forme, qui reflètent en pratique d'importantes divergences culturelles comme le démontre éloquemment Michel Sparer dans son article. Ces différences sont autant de problèmes pour le traducteur en exercice, l'apprenti traducteur et l'enseignant qui a pour mission d'en reconnaître la nature et de proposer des solutions qui évitent les chausse-trappes que tend ce genre d'opération.

\section{Les différences de fond}

Le droit est un peu comme le miroir de la légende, il reflète l'image que nous y projetons mais aussi, en arrière-plan, celle du passé qui se déroule comme un film qui remonterait dans le temps. Ceci pour dire que l'on ne peut raisonnablement espérer cerner le phénomène juridique dans sa manifestation synchronique sans étayer cette observation sur une solide base diachronique.

Le droit que nous pouvons observer à l'œuvre dans tel ou tel pays n'est que le résultat d'une longue et souvent lente (en dehors des accélérations de l'histoire que sont les révolutions) évolution. Il est en cela semblable à l'iceberg dont on ne voit que le dixième de la masse et dont on est porté à sous-estimer l'importance. En l'occurrence, l'important c'est ce que l'on ne voit pas. Comment comprendre les origines du droit des États-Unis ou du Canada sans passer par l'étude du droit anglais, la common law, ou par celle du droit français, le droit civil ? La chose est impossible, les raccourcis trop tentants et forcément sommaires.

Le droit, avons-nous dit, est le reflet d'une certaine conception de l'ordre social. Or, cette conception varie d'un pays à l'autre pour de très nombreuses raisons qui ne sont pas seulement géographiques. Dans l'Esprit des lois, Montes- 
quieu faisait déjà observer que « les lois politiques et civiles de chaque nation [...] doivent être tellement propres au peuple pour lequel elles sont faites que c'est un très grand hasard si celles d'une nation peuvent convenir à une autre ${ }^{30}{ }^{\circ}$.

L'histoire des quatre nations qui nous intéresse ici est trop originale pour avoir engendré un système unique. Si l'on ne peut parler de tronc commun à la common law et au droit civil, on doit cependant admettre une certaine interaction qui va en s'accélérant, au point qu'au rythme actuel il est probable que les différences seront effacées au profit d'un système plus ou moins uniforme qui pourrait prendre la forme d'un «espace juridique commun», comme l'on parle d'un marché commun. L'exemple de la C.E.E. est révélateur à cet égard. Il ne s'agit encore que d'une hypothèse, mais elle prend de plus en plus de relief.

Pour le moment, les divers systèmes affichent encore leurs grandes différences. D'un côté, ce sont les juges qui ont modelé un système dont les fondements sont toujours largement jurisprudentiels parce que le droit anglais pose en principe que le rôle de la règle de droit est de résoudre des litiges, non de les prévoir, selon l'adage bien connu remedies precede rights, la procédure prime le droit.

De l'autre, le droit est un moyen, la justice un but, la règle juridique est * générale » et non «particulière » et l'esprit qui les anime participe d'une conception philosophique, intellectuelle et non purement pragmatique. Il s'agit, pour le droit français, de prévoir et non de "gérer » l'activité sociale.

Ces deux systèmes, en outre, sont issus de deux traditions politiques et philosophiques très différentes, dont l'utilitarisme et le parlementarisme ne sont que deux des manifestations les plus évidentes, alors que le centralisme, l'étatisme, puis l'esprit révolutionnaire ont façonné un droit que ses fondateurs voulaient conforme à la raison. Le résultat, dans les deux cas, est étonnant. D'un côté, on s'attendrait à voir dans le droit anglais le reflet d'une société sereine et sûre d'ellemême, un système logique que des siècles de décisions et de réflexions ont édifié avec une lenteur calculée, donc figé et plutôt conservateur. Or, c'est à ce système que l'on doit l'habeas corpus et autres Bills of Rights, et ce n'est pas la logique mais l'empirisme qui le sous-tend. De l'autre côté, l'observateur peu averti pourrait croire, sur la foi de la tumultueuse histoire de France, construite à coups de révolutions, de conflits et de guerres, ainsi qu'à grand renfort de constitutions, que le droit d'un tel pays est instable, irrationnel, à l'image de son peuple, quand c'est le contraire qui est vrai et que le moule fragile et informe a produit un archétype de logique et de rigueur classique, un code de référence quasi universel et un modèle de stabilité. Il ne faut donc pas s'étonner qu'ils aient donné naissance, sur la base d'institutions aussi différentes, à deux régimes qui se caractérisent par l'administration judiciaire des pays anglo-saxons et par le régime administratif du droit français.

La vieille distinction droit écrit — droit non écrit s'estompe peu à peu. L'exemple des États-Unis est significatif à cet égard, la loi y occupant une place

30. De l'Esprit des Loix, 1748, 1, 3. 
privilégiée. C'est également le cas du Canada dont les législateurs s'inspirent de plus en plus de l'exemple américain, au détriment de l'influence britannique.

Il reste que des principes différents ont façonné des notions particulières diffcilement transposables d'un système dans l'autre. On connaît les difficultés que présentent, pour le traducteur, des termes dont il n'existe pas d'équivalent dans l'autre culture juridique, à commencer par common law et equity. Et que dire des termes de procédure, nombreux et originaux, particuliers au droit anglais ? A-t-on jamais trouvé un équivalent satisfaisant pour due process, trust/trustee/trustor et beaucoup d'autres encore? Et inversement, quelles notions de droit anglais recouvrent précisément le droit des obligations, façon code civil ? Le fait d'attribuer un « équivalent \$ à un mot pour lequel il n'existe pas de notion comparable dans l'autre système peut prêter à confusion et entraîner parfois de graves erreurs en raison des effets qu'il véhicule.

Mais les difficultés que présente la traduction juridique ne se posent pas uniquement sur le plan du fond et de la terminologie, et la façon dont elles sont résolues dans la rédaction du texte de LA ne présente bien souvent que peu de rapports avec l'économie, la syntaxe de cette langue. C'est un des gros problèmes que pose cette forme de traduction.

\section{Les difficultés de la rédaction juridique}

Comme cette question est évoquée à plusieurs reprises dans le présent numéro, nous nous contenterons de la présenter dans ses grandes lignes.

Chaque langue suit, dans son expression écrite, un schéma d'organisation et de fonctionnement qui lui est propre. La langue juridique française passe, à tort ou à raison, pour être claire, précise et concise, ce que devrait être tout langage du droit puisqu'il est destiné, au premier chef, à tout le monde et devrait pouvoir être compris du plus grand nombre. Tel n'est pas toujours le cas mais il reste que l'ambiguité doit être éliminée et l'interprétation, la moins équivoque possible. À moins que, comme dans le cas de certains textes d'accords internationaux, l'ambiguïté n'ait été volontairement introduite pour des raisons diplomatiques ${ }^{31}$.

En règle générale, il n'en va pas ainsi et le texte juridique de langue française suit une logique d'exposition qui est le produit d'une longue tradition universitaire qui a trouvé son épanouissement dans le siècle des lumières et s'est concrétisée dans la codification des textes de loi français, notamment le célèbre Code civil de 1804. Un bon exemple, celui de l'article 2 du Code civil, illustrera ce propos : «La loi ne dispose que pour l'avenir ; elle n'a point d'effet rétroactif. \$

La formule est claire, sans équivoque, quoique lapidaire. Qu'en est-il du côté de la langue anglaise et de sa forme d'expression écrite? Un simple exemple suffira à nous éclairer sur ce point. Elliott Biskind rapporte qu'en 1969 les cours suprêmes de l'État de New York entendirent 2260 causes portant sur des questions contractuelles. Sur ce nombre, la moitié tournaient autour de problèmes d'interprétation ${ }^{32}$.

31. C'est le cas de la célèbre résolution des Nations unies de 1967 et de son interprétation des mots withdrawal from occupied territories que l'on peut comprendre de deux façons.

32. Elliott L. Biskind, Simplify Legal Writing, op. cit., p. 4. 
Cet état de choses n'est pas seulement l'indice d'une détérioration du langage, il tient au fait que le style de rédaction juridique anglaise se distingue du type de rédaction courante comme nous l'expliquent Myers et Jones :

Yet, while the layman conventionnally speaks in a right-branching fashion, the lawyer very often makes his sentences using left-branching constructions. In Access to the Law, Dean Friedland suggests that the lawyers' overuse of left-branching sentences in legal discourse is rooted in history. He notes the antiquated rules of drafting written by George Coode in 1843 called On Legislative Expression. There, Coode states that good legislative style requires that the conditions be stated before the thing that the conditions apply to. Even today, notes Friedland, "Coode's work is still regarded as a guide to good drafting ${ }^{33}$.

Il s'ensuit que la rédaction juridique anglaise ne peut et ne doit ni inspirer ni influencer le traducteur car elle ne suit pas les mêmes critères d'exposition que ceux qui ont été établis pour la langue française et engendre des ambiguités contre lesquelles celle-ci n'a pas les moyens de lutter efficacement. La logique doit l'emporter dans tous les cas, quitte à modifier au besoin l'ordonnance des phrases pour rendre un énoncé plus clair pour l'utilisateur francophone. Trop de traducteurs se laissent influencer par la structure anglaise et, soit par ignorance, soit par négligence, ont tendance à la calquer et à la reproduire dans leurs traductions. Ce phénomène n'est pas particulier à la langue française comme en témoignent les textes des Nations unies traduits de l'anglais vers d'autres langues. Ce faisant, ces traducteurs pèchent contre la clarté et la précision de leur propre langue et contribuent à l'abâtardir.

Les deux difficultés que représentent l'absence d'une terminologie fondamentale et les différences de fond et de forme que présentent les systèmes juridiques posent le problème de la documentation.

\section{LE TRADUCTEUR JURIDIQUE ET LA DOCUMENTATION}

De tous les problèmes que le traducteur est amené à résoudre, dans l'exercice quotidien de sa profession, celui de la documentation est sans doute le plus délicat.

Or, c'est dans le domaine juridique que cette question se pose avec le plus d'acuité car, contrairement au traducteur spécialisé dans le secteur financier ou technique, le traducteur juridique doit surmonter l'obstacle dont nous avons parlé plus haut, soit rendre dans LA des notions ou des réalités inconnues de celle-ci parce qu'elles ne reposent sur aucun dénominateur commun aux deux systèmes en présence.

Le langage de la technique ou de la finance peut varier d'un pays à l'autre mais les phénomènes qu'il décrit sont identiques; les lois de la physique ou les mécanismes de la Bourse sont les mêmes à Londres, à Tokyo, à New York ou à Paris et les mouvements de capitaux, à l'ère multinationale, ignorent les frontières.

Tel n'est pas le cas de la règle juridique pour laquelle les frontières constituent souvent un obstacle infranchissable puisqu'elle ne s'applique que dans le cadre limité de l'État qui l'a conçue. Cela ne manque pas de compliquer singu-

33. E.R. Myers et C.S. Jones, Language and Jury Instructions, op. cit. 
lièrement la tâche du traducteur, notamment de celui qui exerce en milieu bilingue comme au Canada où cohabitent deux systèmes juridiques s'inspirant de sources différentes, le droit civil au Québec et la common law dans tout le reste du Canada.

C'est dire la place que tient la documentation pour le traducteur juridique constamment tenu de puiser à 3 ou 4 sources différentes les renseignements qui lui permettront de faire le lien entre les deux systèmes en présence, dont les origines ne sont pas toujours aussi claires que la rigueur et le caractère obligatoire de la loi pourraient porter à croire. Au contraire, il peut sembler paradoxal d'avancer que le droit est un des rares domaines où la documentation soit aussi peu fiable, alors que le volume annuel des publications et, surtout, des décisions, dépasse parfois l'entendement (comme aux Etats-Unis). Ce serait oublier qu'un système juridique est difficilement transposable dans un autre car, compte tenu des raisons exposées plus haut, il n'y a pas nécessairement équivalence entre les deux. Le droit actuel des États-Unis, par exemple, ne présente que de lointains rapports avec le droit anglais qui en est la source principale; le système juridique en vigueur au Québec, façonné par les institutions parlementaires et le droit britanniques, présente moins de ressemblances que de différences avec la famille romano-germanique; le Canada et les Etats-Unis, dont les systèmes juridiques se rapprochent insensiblement, s'éloignent de plus en plus du modèle et du tuteur britanniques.

L'opération de documentation, pour le traducteur, commence généralement par les dictionnaires, unilingues d'abord puis bi- ou multilingues, qui vont l'orienter vers la solution. Mais quelle solution choisir dans un domaine qui se caractérise par une grande abondance de termes, une polysémie chronique et une synonymie non moins importante? Quand, en outre, cette terminologie est difficilement « exportable \$ puisque la réalité juridique d'un pays ne peut être impunément calquée sur celle du voisin en raison des différences socio-culturelles et socioéconomiques qui se reflètent dans les institutions?

C'est sur cette difficulté, simple à comprendre en théorie mais d'application si complexe, que viennent régulièrement buter depuis plus de 60 ans les appels lancés en faveur de l'élaboration d'une linguistique juridique ${ }^{34}$. Tous les auteurs qui se sont attaqués à ce problème signalent invariablement l'absence d'une tentative concertée de lexicographie juridique "différentielle » qui tiendrait compte à la fois de la réalité juridique et des impératifs linguistiques.

La production dans ce domaine est faible. Le traducteur se trouve soit en présence de (bons mais rares) dictionnaires juridiques unilingues établis par des juristes de grande réputation (Capitant, Black, entre autres), soit en présence de dictionnaires de traduction bilingues, les meilleurs généralement, ou multilingues, les plus faibles et les moins fiables.

\section{Le besoin d'une lexicographie juridique}

Le besoin de bons outils de travail dans le domaine juridique ne date pas d'hier. En 1967, les conclusions et recommandations des participants au stage

34. Voir François Gény, cite par Focsaneanu, op. cit., MIe partie, p. 448 et nos 256 et 257. 
sur les langues de spécialité organisé à Saint-Cloud reconnaissaient la nécessité de créer un centre européen de terminologie juridique et économique ayant pour objectif de recenser le vocabulaire de ces spécialités et de le définir, afin de réaliser des dictionnaires multilingues de terminologie juridique ${ }^{35}$. Depuis, chaque étude ou presque portant sur la terminologie ou le langage du droit signale l'urgence du problème et la complexité des solutions possibles ${ }^{36}$.

Il serait trop long d'exposer ici en détail les raisons d'une telle situation. Du strict point de vue de l'utilisateur (enseignant, traducteur, terminologue, étudiant), le problème se ramène à trois critères principaux : l'exhaustivité, la fiabilité et l'utilité des dictionnaires disponibles dans un domaine. Tel est le souci qui a présidé à l'élaboration des premières bibliographies de ce type destinées à répondre plus directement aux besoins du traducteur spécialisé ou non ${ }^{37}$.

On sait que l'exhaustivité plus ou moins grande d'un dictionnaire (et autres ouvrages du genre) atteste du sérieux et de la qualité des recherches faites par le ou les auteurs, à condition de ne pas confondre l'exhaustivité du vocabulaire d'un domaine, sa terminologie, et l'exhaustivité encyclopédique et tentaculaire qui s'approprie les termes des disciplines connexes sous couvert de connotation, fût-elle juridique. On touche ici au cour du problème des dictionnaires de traduction qui font trop souvent profession de fournir des « recettes » au détriment de solutions parce qu'ils sont généralement établis à partir de traductions et non de textes originaux. Tel est notamment le cas des dictionnaires multilingues dont les lacunes sont flagrantes, sauf dans la langue (ou les deux langues) qui a servi de modèle. Cette faiblesse a trop souvent été dénoncée pour que nous nous y attardions; le danger d'une solution s'inspirant d'une traduction est grand et celle-ci ne devrait servir que d'indication, d'élément de réponse susceptible d'orienter l'utilisateur. Mais l'exhaustivité est, là encore, relative et un lexique de 1500 termes peut être assez complet alors qu'un dictionnaire de 10000 entrées peut paraître, en comparaison, peu exhaustif, ce critère étant fonction du domaine auquel il s'applique. A titre d'exemple, le vocabulaire de la météorologie, comparativement à celui de l'èconomie ou du droit, est peu abondant.

La fiabilité prend en compte la qualité linguistique des entrées, définitions et équivalents proposés, ainsi que le degré de vieillissement de la terminologie, mal dont souffrent de très nombreux ouvrages. Elle rejoint les préoccupations évoquées à propos de l'exhaustivité auxquelles s'ajoutent le souci de la présentation, la clarté de l'énoncé, la concision et la pertinence des définitions, et d'autres critères encore. La combinaison « juriste-linguiste » prend ici toute sa signification. L'idéal de la lexicographie juridique tient dans ces deux conditions : aux

35. Actes du stage de Saint-Cloud, 23-30 nov. 1967, publiés sous le titre les Langues de spécialité, analyse linguistique et recherche pédagogique, AIDELA, Strasbourg, 1970, p. 348 .

36. Voir en particulier la communication de $\mathbf{M}$. Doucet sur « l'inventaire des dictionnaires en matière juridique ", Actes du stage de Saint-Cloud, op. cit., p. 256 et suiv.; J.-L. Sourioux et P. Lerat, le Langage du droit, op. cit., p. 89 et suiv.; J. Le Tellier, Q Quelques thèmes de réflexion en vue de la rencontre franco-québécoise de linguistique appliquée (langue juridique) $\gg, 25$ sept. - 1er oct. 1977 (document non publié).

37. J.-C Gémar et Paul A. Horguelin, Bibliographie sélective du traducteur - Commerce et économie, Montréal, Linguatech, 1977,180 p.; J.-C. Gémar, Bibliographie sélective du traducteur - Droit et justice, Montréal, Linguatech, $1978,280 \mathrm{p}$. 
connaissances du domaine de spécialité le lexicographe devrait ajouter celles de la linguistique s'il veut atteindre un niveau optimal de qualité.

L'utilité, enfin, dépend dans une large mesure des deux critères précédents dont elle constitue la résultante en quelque sorte. Elle est proportionnelle au degré d'exhaustivité et de fiabilité du dictionnaire. Ce critère est également destiné à renseigner l'utilisateur sur le niveau et la qualité du service qu'il est en droit d'attendre d'un ouvrage de référence, compte tenu de ses besoins particuliers, notamment du contexte juridique et linguistique de LA. Par exemple, un glossaire multilingue à l'usage des Communautés européennes peut induire en erreur l'apprenti traducteur qui ignorerait la nature particulière des textes de droit communautaire. Et vice versa.

Cet aspect du problème de la documentation en droit ne représente qu'une des multiples facettes de la question car, dans ce domaine, les dictionnaires ne constituent qu'un des outils de travail du traducteur ${ }^{38}$ et les recherches, au Canada, aux États-Unis et en Europe vont bon train. Les décisions des tribunaux dans les pays où la jurisprudence tient une place importante, l'accès à celle-ci et leur diffusion jouent également un grand rôle comme en témoignent les efforts d'automatisation de l'information que consentent de nombreux pays, dont le Canada et la France.

Cette dernière considération témoigne du rôle de plus en plus grand de l'interdisciplinarité dans la formation et l'apprentissage de la traduction juridique dont l'unité repose sur une base de connaissances qui puisent largement dans les préoccupations communes à plusieurs disciplines.

\section{L'APPROCHE PLURIDISCIPLINAIRE DE LA TRADUCTION JURIDIQUE}

Nous savons déjà que le traducteur juridique doit avoir reçu une double formation (juridique et linguistique) s'il veut espérer surmonter les difficultés que présente cette discipline et éviter les pièges que pose l'opération de transfert. Nous pourrions même aller jusqu'à dire que plus l'éventail des disciplines connues de lui est ouvert, plus grandes sont les chances d'appréhender avec succès la solution du problème posé.

Une approche interdisciplinaire de la traduction juridique semble être la solution la plus logique, la plus pratique. Mais par interdisciplinarité il ne faut pas seulement entendre l'histoire, la linguistique ou les sciences politiques. L'économie, la philosophie, la sociologie et, éventuellement, la théologie (le droit canon) et la psychologie sociale sont autant de disciplines dont l'absence pourrait fausser le jugement d'une personne non avertie. Cette liste n'est sûrement pas exhaustive.

Comment, en effet, envisager sérieusement d'étudier le droit commercial ou fiscal sans recourir à de solides notions d'économie politique? La criminologie ne peut être dissociée du droit, pas plus que de la psychologie et de la médecine, pour qui veut maîtriser cette vaste discipline. Quant à la sociologie, comment

38. Voir sur ce point l'importante étude faite par André Dunes, Documentation juridique, Paris, Dalloz, 1977, 198 p. 
pourrait-on ne pas en tenir compte dès lors qu'il est question de normes sociales, de sciences sociales et humaines?

En outre, les contacts interdisciplinaires sont, à l'évidence, très enrichissants surtout lorsqu'ils ont l'histoire comme dénominateur commun parce que la connaissance des systèmes et des institutions d'autrefois nous aide à mieux comprendre ceux d'aujourd'hui. Comment, en effet, parvenir à comprendre le droit des EtatsUnis ou du Canada sans remonter aux sources du droit anglais? Ou encore, comment saisir l'esprit du droit français et de la famille romano-germanique sans passer par l'étude de l'organisation politique des Grecs et des Romains, et de leurs cités. La conscience de la relativité du droit ne peut venir que d'une comparaison objective des milieux, des sociétés et des cultures à travers les âges; l'on ne peut suivre l'évolution du concept de règle juridique si l'on dissocie l'étude du droit de celle de la morale et de la religion car la laïcisation ne s'est pas réalisée en un jour et la théorie du droit «naturel » est loin d'avoir rallié tous les suffrages. Nombreux sont encore les systèmes juridiques fondés sur une religion (entre autres, le droit musulman et le droit hindou), bien que l'autonomie du droit gagne de plus en plus de terrain dans la plupart des pays et que les sciences juridiques s'orientent peu à peu vers un système de morale universelle (les droits de l'homme par exemple) qui participe du droit naturel.

De telles contraintes, liées à l'enseignement de cette discipline autonome qu'est en passe de devenir la traduction juridique, posent de gros problèmes de méthodologie. La formation du traducteur est au centre du débat car comment concilier, en un ou deux cours de traduction juridique tous les impératifs évoqués plus haut quand on sait qu'il faut 3 ou 4 années bien remplies pour former un juriste ? Simone Dreyfus apporte un élément de réponse en faisant la distinction entre " l'enseignement visant l'acquisition d'une connaissance passive de la langue spécialisée 》 et l'enseignement qui vise «à l'acquisition d'une connaissance active de la langue spécialisée ${ }^{30}{ }^{~}$. Dans le premier cas, l'essentiel pour l'étudiant est bien de comprendre et de pouvoir ensuite traduire. Mais pour ce faire, encore faut-il qu'il soit en mesure de "comprendre », qu'il ait reçu une formation secondaire et même universitaire qui l'ait préparé à aborder plusieurs domaines différents et inconnus de lui. L'orientation actuelle des études avec le système du choix des cours «à la carte 》 ne prédispose pas toujours à ce genre d'expérience, bien qu'il ait été conçu pour encourager l'interdisciplinarité.

La traduction juridique, pourtant, implique une parfaite connaissance générale du sujet, du moins à un certain niveau de problèmes. On ne peut se contenter d'enseigner des mots et ignorer les concepts qu'ils recouvrent. Selon Vinay, « pour traduire certains textes techniques portant sur [...] le droit international, il vaut mieux s'adresser à [...] des juristes sachant l'anglais, le français ou l'espagnol ${ }^{40} \gg$. C'est ce que pensent beaucoup d'employeurs et de juristes qui tiennent le même raịsonnement et répugnent à confier des travaux de nature juridique aux traducteurs. La réalité se situe sans doute ailleurs, au niveau des personnes ayant reçu la

39. Actes du stage de Saint-Cloud, op. cit., p. 222-223. C'est moi qui souligne.

40. Journal des traducteurs, op. cit., p. 144. 
double formation qui garantit un minimum de qualité linguistique et une connaissance certaine du domaine.

Une approche pluridisciplinaire est donc nécessaire pour faire de la traduction dans le domaine juridique, faute de quoi l'apprenti traducteur s'expose à bien des déconvenues car une chose est de traduire des mots, une autre d'en comprendre toute la portée sur la base de solides connaissances diachroniques des données d'un problème généralement posé en termes synchroniques. L'idéal consisterait à trouver une formule logique qui s'appliquerait automatiquement dans la plupart des cas et qui garantirait l'intégrité de la règle juridique. Un tel objectif, pour le moment du moins, est difficile à atteindre car la diversité des systèmes en présence et le nombre incalculable de normes qu'ils véhiculent sont trop grands. En outre les sciences juridiques, n'ayant pas vocation « nomothétique s, selon la distinction de Piaget; la mise en équation du problème se heurte à un nombre d'inconnues encore trop élevé.

Une esquisse de formule logique pourrait néanmoins servir de conclusion à notre démonstration. L'opération de traduction juridique $\left(T_{\mathfrak{f}}\right)$ suit une série d'étapes et subit un certain nombre de contraintes entre le départ, $L D$, et l'arrivée, LA. Elle dépend au moins, dans chacun des cas, des inconnues que sont la terminologie, son champ sémantique $\left(T_{s}\right)$ et la documentation $(D), T_{s}$ et $D$ étant fonction du caractère normatif du droit $\left(\mathrm{N}_{\mathrm{d}}\right)$ et s'inscrivant dans un ensemble qui représente les systèmes juridiques $\left(S_{1}, S_{2}, \ldots\right)$ en présence. Soit :

$$
\begin{aligned}
& T_{i}=f(L D, L A) \\
& L D=f_{1}\left(T_{s}, D, N_{d_{1}}, S_{1}, \ldots\right) \\
& L A=f_{2}\left(T_{s}, D, N_{d_{2}}, S_{2}, \ldots\right)
\end{aligned}
$$

Nous ne prétendons pas avoir mis en équation le problème de la méthodologie de la traduction juridique parce qu'il reste encore trop d'inconnues et que notre démarche est exploratoire. Elle se veut cependant pratique et peut servir de point de départ à l'établissement d'une véritable méthodologie. Nous croyons néanmoins que toute approche devrait s'inspirer d'une forme de logique juridique, seul facteur essentiel de la marche épistémologique parce qu'il part d'un fait établi, celui de la réalité du droit et passe par la méthodologie qui représente le moyen terme entre la pratique et la théorie.

Quelle que soit la solution envisagée, il ne faudra pas perdre de vue les conseils que Marouzeau donne aux traducteurs :

La traduction est nécessairement une lutte. Le bon traducteur est celui qui cherche, qui se pose des questions, qui, loin de se contenter de ce qu'il a trouvé d'abord, commence par s'en méfier; il est comme le médecin scrupuleux qui, son diagnostic à peine posé, cherche les indices qui pourraient le conduire à le remplacer par un autre mieux fondé. En matière de traduction, on pourrait dire que la première idée n'est jamais la meilleure ${ }^{41}$.

Le traducteur juridique, encore plus que les autres, est engagé dans cette lutte dont il sort parfois difficilement vainqueur.

JeAn-Claude Gémar

41. La traduction du latin; conseils pratiques, Paris, Les Belles Lettres, 1951, p. 74. 